\title{
On Subjective and Objective Quality Evaluation of Point Cloud Geometry
}

\author{
Evangelos Alexiou and Touradj Ebrahimi \\ Multimedia Signal Processing Group (MMSPG) \\ École Polytechnique Fédérale de Lausanne (EPFL) \\ CH-1015, Lausanne, Switzerland \\ Emails: FirstName.LastName@epfl.ch
}

\begin{abstract}
Point clouds have emerged as a promising solution for immersive representation of $3 \mathrm{D}$ contents. In this paper an interactive subjective quality assessment for point clouds is proposed. Quality assessment of geometry information in point clouds subject to realistic types of degradations is performed and correlation between state-of-the-art objective metrics and ground truth subjective scores is investigated. Preliminary results suggest that there is a need for more appropriate objective metrics, since the current solutions are not able to provide accurate predictions of quality for every type of degradations and contents.
\end{abstract}

Keywords—point clouds; quality assessment; quality metrics

\section{INTRODUCTION}

Point clouds are commonly used for storage and rendering of 3D models in virtual, augmented and mixed reality, photogrammetry and 3D printing applications. A point cloud could be interpreted as a collection of three-dimensional points in space representing the external surface of an object. Each sample is defined by its position, which is obtained by its measured or reconstructed X, Y, and Z coordinates. Associated features, called attributes, are also often used in conjunction with the coordinate data to provide further information.

Quality and user experience are extremely important factors in visual communication. The quality is, typically, assessed via either subjective or objective methods. In objective evaluation of point clouds geometry, similarity is the key factor. The stateof-the-art objective metrics for geometric distortions can be classified as point-to-point (p2point), point-to-plane (p2plane) or point-to-mesh [1], [2], [3]. Point-to-mesh distances depend on surface reconstruction techniques used for the mesh, hence, they are considered as suboptimal.

A limited amount of work on subjective assessment of point cloud data has been reported in the literature. In [4], a $3 \mathrm{D}$ tele-immersive system is proposed and users represented by their avatars are able to interact in a virtual room. In [5], different resolutions and values of geometric and color noise in point clouds are assessed. The uniform noise that is considered, though, does not correspond to a realistic model, neither for geometric nor for color degradations of point clouds. In both cases, the p2plane metrics are not examined and the correlation between objective and subjective metrics is not reported.

This paper shows that current state-of-the-art objective metrics do not predict well visual quality, especially under typical distortions such as compression.

QoMEX2017 - Erfurt, Germany; 978-1-5386-4024-1/17/\$31.00 @2017 IEEE

\section{Testing Data set Preparation}

The geometry of five point clouds under two different types of degradations is assessed in this experiment; thus, simple scenes and objects normalized in the dynamic range of $[0,1]$ are selected. Under this context, bunny (35947 points) and dragon (437645 points) from Stanford 3D Scanning Repository ${ }^{1}$ represent existing and widely used point clouds. Cube (30246 points) and sphere (30135 points) are artificially generated and represent synthetic contents. Finally, vase (108587 points), a model captured by Intel RealSense R200, is used. Values of parameters to define distortions were selected to represent subjective quality covering a wide range from lowest to highest levels.

\section{A. Noise}

Gaussian noise models position errors due to imperfections in acquisition. This model is widely used in the literature. The noise affects all points of the point cloud and its level is determined by a target standard deviation (i.e., $\sigma=\{0.0001,0.0005,0.002,0.008\}$ ).

\section{B. Octree-pruning}

Octree structure is extensively adopted in point cloud compression algorithms as it enables organized representation of points. This regular representation, though, leads to visible artifacts in the form of structured distortions. Octree-pruning can be obtained by setting a desirable octree resolution; this defines the size of leaf nodes, the center of which replaces the appended points. It could be interpreted as an instance of progressive decoding procedure, as it creates similar distortions. Octree resolution values are properly selected for each content to obtain target percentages $(p)$, with respect to the original number of points (i.e., $p=\{30 \%, 50 \%, 70 \%, 90 \%\}$ ).

\section{Subjective Evaluations}

To display the stimuli, PCLVisualizer [6] with an Apple Cinema Display of 30 inch (res. 2560x1600) was used. The subjects were able to interact with the content by composite movements of mouse (left, right, up, down, zoom) and provide their score using keyboard, with no time restriction. The double-stimulus impairment method with 5-scale rating was selected. The original and the processed contents were

\footnotetext{
${ }^{1}$ http://graphics.stanford.edu/data/3Dscanrep/
} 
displayed and rotated simultaneously, resulting in a side-byside visualization. The reference was clearly indicated, and the same content never appeared consecutively. Different permutations of the position of the original and processed contents were applied to each subject. The evaluation procedure included two sessions (one for each degradation), with a training phase. For each of the 5 contents, 4 degradation values were used along with a hidden reference, leading to 25 stimuli per session. A total of 17 naïve subjects between 25 and 36 years old ( 9 females and 8 males, avg $=28$ years) participated.

\section{ObJective Metrics \& Performance IndeXes}

Geometric errors between the original and the processed point clouds can be estimated either using the root mean square (RMS) difference or the Hausdorff $(\mathrm{H})$ distance for both p2point and p2plane cases. The p2point error is calculated by connecting each point of the reference to the closest point of the processed point cloud. The p2plane error measures the projected error along the normal of the original point [3]. Commonly, the symmetric distance is used; that is, obtained by setting both the original and the processed content as reference and estimate both errors. Then, the maximum value is considered. However, such absolute values of error fail to assess differences between differently scaled contents. For this purpose, PSNR ratio seems to be a more appropriate metric. In the literature, it is defined as the ratio of the maximum distance of the nearest neighbours, or the distance of the diagonal of the bounding box divided by the error (i.e., either RMS or H).

Subjective Mean Opinion Scores (MOS) are used as ground truth. Following [7], the result of a particular objective metric is called Point cloud Quality Rating (PQR). A cubic function is fitted to each [PQR, MOS] pair providing a predicted MOS value, denoted as $\operatorname{MOS}_{P}$. Then, Pearson linear correlation coefficient (PCC), Spearman rank order correlation coefficient (SCC), root-mean-square error (RMSE) and outlier ratio (OR) were computed between MOS and $\mathrm{MOS}_{P}$ to estimate linearity, monotonicity, accuracy and consistency, respectively.

\section{TABLE I. PERFORMANCE INDEXES.}

\begin{tabular}{|c|c|c|c|c|c|c|c|c|}
\hline & \multicolumn{4}{|c|}{ Gaussian noise } & \multicolumn{4}{|c|}{ Octree-pruning } \\
\hline Metric: & PCC & SCC & RMSE & OR & PCC & SCC & RMSE & OR \\
\hline p2point ${ }_{\text {RMS }}$ & 0.987 & 0.917 & 0.223 & 0.15 & 0.371 & 0.227 & 0.868 & 0.70 \\
\hline p2plane $\mathrm{RMS}$ & 0.991 & 0.942 & 0.188 & 0.15 & 0.295 & 0.274 & 0.893 & 0.65 \\
\hline p2point $_{H}$ & 0.994 & 0.983 & 0.151 & 0.10 & 0.439 & 0.292 & 0.840 & 0.65 \\
\hline p2plane ${ }_{H}$ & 0.993 & 0.970 & 0.162 & 0.10 & 0.419 & 0.254 & 0.849 & 0.65 \\
\hline PSNR_p2 point ${ }_{\text {RMS }}$ & 0.991 & 0.990 & 0.187 & 0.15 & 0.442 & 0.272 & 0.839 & 0.60 \\
\hline PSNR_p2plane ${ }_{R M S}$ & 0.981 & 0.979 & 0.266 & 0.15 & 0.429 & 0.275 & 0.844 & 0.60 \\
\hline PSNR_p2point $_{\mathrm{H}}$ & 0.988 & 0.979 & 0.213 & 0.10 & 0.469 & 0.347 & 0.826 & 0.50 \\
\hline PSNR_p2plane $_{\mathrm{H}}$ & 0.987 & 0.981 & 0.221 & 0.15 & 0.453 & 0.320 & 0.834 & 0.55 \\
\hline
\end{tabular}

\section{Results}

In Fig. 1 and Fig. 2, the scatter plots of subjective scores against the most efficient objective metric is presented for noise and octree-pruning. As can be observed, our results show strong correlation for Gaussian noise. Considering that the objective metrics rely on the distance between the original and the processed contents, by increasing the standard deviation of the noise, the obtained results worsen. Moreover, since geometryonly point clouds are visualized side-by-side, subjects could easily identify the amount of noise in the processed stimuli.

However, there is no good correlation when compressionlike artifacts are introduced. The octree-pruning significantly affects the perceived quality of curved surfaces, and this degradation is decently captured by objective metrics. Similar errors are also obtained for cube. Subjective scores indicate, though, that in this case, quality degradation is less critical due to the particular geometric nature of this content which leads to less visible distortions for content with planar structures, such as cube.

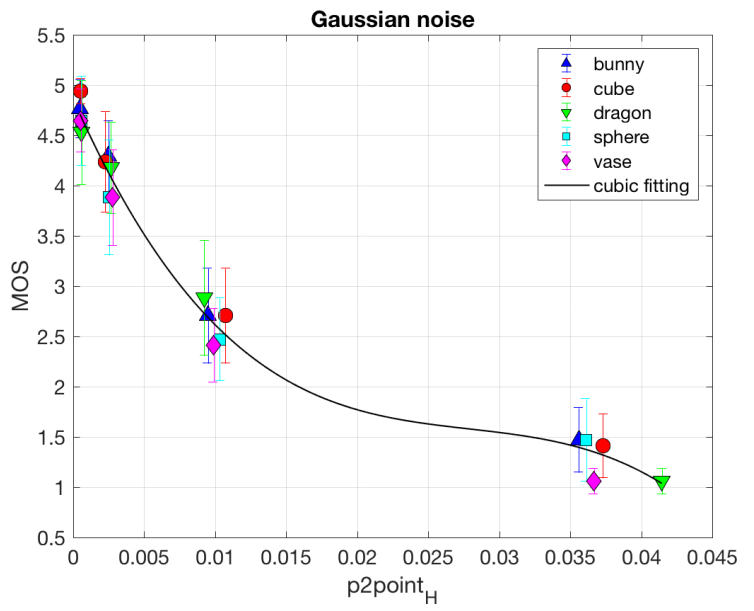

Fig. 1. Subjective vs objective results in the presence of Gaussian noise.

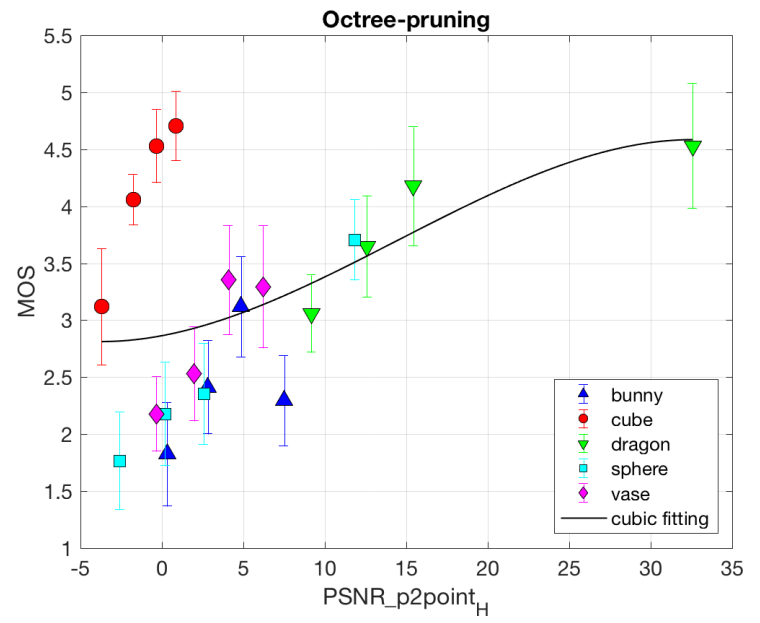

Fig. 2. Subjective vs objective results after octree-pruning.

\section{CONCLUSION}

Our results show that state-of-the-art objective metrics perform well in the presence of Gaussian noise. However, they fail to always correctly predict the perceptual quality of compression-like distortions. Performance to predict distortions due to octree-pruning seems to be content dependent, which indicates that there is a need for better objective metrics that can more accurately predict all practical types of distortions and contents.

\section{ACKNOWLEDGEMENT}

This paper reports a research performed under the framework of a collaborative project Random Access Video Encoding (RAVE) with Teleport SA and funded by The Swiss Commission for Technology and Innovation (CTI) under the grant 18875.1PFES-ES. 


\section{REFERENCES}

[1] P. Cignoni, C. Rocchini, and R. Scopigno, "Metro: Measuring Error on Simplified Surfaces," Computer Graphics Forum, vol. 17, no. 2, pp. 167174, Jun. 1998.

[2] R. Mekuria, Z. Li, C. Tulvan, and P. Chou, "Evaluation criteria for point cloud compression,” ISO/IEC MPEG n16332, Geneva, Switzerland, February 2016.

[3] D. Tian, H. Ochimizu, C. Feng, R. Cohen, and A. Vetro, "Evaluation metrics for point cloud compression," ISO/IEC JTC m74008, Geneva, Switzerland, January 2017.

[4] R. Mekuria, K. Blom, and P. Cesar, "Design, Implementation and Evaluation of a Point Cloud Codec for Tele-Immersive Video," IEEE Transactions on Circuits and Systems for Video Technology, pp. 1-1, 2016.

[5] J. Zhang, W. Huang, X. Zhu, and J. N. Hwang, "A subjective quality evaluation for 3D point cloud models," in 2014 International Conference on Audio, Language and Image Processing, Jul. 2014, pp. 827-831.

[6] R. B. Rusu and S. Cousins, "3D is here: Point Cloud Library (PCL)," in 2011 IEEE International Conference on Robotics and Automation, May 2011, pp. 1-4.

[7] ITU-T P.1401, "Methods, metrics and procedures for statistical evaluation, qualification and comparison of objective quality prediction models," International Telecommunication Union, July 2012. 